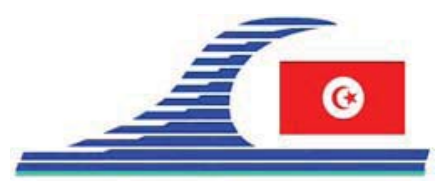

\author{
Conférence Méditerranéenne Côtière et Maritime \\ EDITION 1, HAMMAMET, TUNISIE (2009) \\ Coastal and Maritime Mediterranean Conference \\ Disponible en ligne - http://www.paralia.fr-Available online
}

\title{
Traitement d'un sédiment portuaire contaminé par bio- remédiation et stabilisation géochimique
}

\author{
Yannick MAMINDY-PAJANY ${ }^{1,2}$, Charlotte HUREL ${ }^{1}$, \\ Nicolas MARMIER ${ }^{1}$, Michèle ROMÉO ${ }^{2}$
}

1. Laboratoire de Radiochimie, Sciences Analytiques et Environnement, Université de Nice Sophia Antipolis, 28 avenue Valrose, Faculté des Sciences, 06108 Nice Cedex 02, France.

mamindy@unice.fr;hurel@unice.fr;marmier@unice.fr

2. Laboratoire des Ecosystèmes marins côtiers et réponses aux stress, Université de Nice Sophia-Antipolis (ECOMERS/EA 4228), France.

romeo@unice.fr

\section{Résumé :}

Le devenir des sédiments est problématique dans certaines régions françaises en raison d'une contamination significative en polluants organiques et/ou minéraux. Dans cette étude, un sédiment de dragage portuaire a été traité par compostage et stabilisation géochimique. Après caractérisation de la contamination (teneurs totales en polluants organiques et minéraux), des andains de $6 \mathrm{~kg}$ de sédiment ont été traités par différents additifs minéraux en présence d'un sédiment témoin en milieu extérieur. Les résultats montrent que (i) les flux de polluants sont réduits de moitié dans les sédiments traités à l'hématite et au fer zéro (ii) la zéolithe fine (clinoptilolite) est peu efficace pour la rétention des polluants dans le sédiment (iii) le compostage permet de réduire entre 38 et $91 \%$ des teneurs en organoétains et $15 \%$ des HAPs.

\section{Mots clés :}

Sédiment de dragage - Stabilisation - Compostage - Additifs minéraux

\section{Introduction}

Les zones côtières sont le lieu d'activités multiples, parmi lesquelles la navigation tient une place importante. L'accès aux structures portuaires est maintenu par des opérations de dragage pratiquées dans les chenaux et dans les bassins de stationnement. En moyenne 50 millions de $\mathrm{m}^{3}$ de sédiments sont dragués chaque année dans les principaux ports français. Le devenir de ces sédiments est problématique dans certaines régions françaises en raison d'une contamination significative en polluants organiques et/ou minéraux. Au niveau national, la gestion des sédiments de dragage s'appuie sur l'arrêté du 14 juin 2000 qui fixe les valeurs guides («niveaux GEODE ») pour les métaux lourds et les PCB (polychlorobiphényles), (ALZIEU et al., 1999). Ainsi, lorsque les concentrations en polluants contenus dans les sédiments marins sont supérieures aux DOI: $10.5150 / \mathrm{cmcm} .2009 .032-9$ 
niveaux GEODE N2 leur immersion est interdite. Depuis 2002, la classification des déchets positionne les boues de dragages dans la catégorie des déchets de construction et de la démolition. Cette nouvelle approche impose aux autorités portuaires de prendre en compte les objectifs de la loi sur les déchets et notamment de réduire la nocivité des déchets et de les valoriser.

Dans cette étude, un sédiment de dragage portuaire a été traité par compostage et stabilisation géochimique. Le compostage actif des sédiments consiste à humidifier et à aérer le sédiment chaque semaine afin de favoriser la dégradation des polluants organiques. Cette bio-remédiation a été associée à un traitement par stabilisation géochimique en ajoutant une phase minérale au sédiment contaminé dans le but de piéger les polluants et de limiter ainsi leur dispersion dans l'environnement. Le sédiment a été composté en présence de différents additifs minéraux (hématite, fer métallique et zéolithes (clinoptilolite) sur une période de 3 mois. Au cours de cette bioremédiation, les eaux de percolation ont été collectées lors de chaque phase d'humidification hebdomadaire afin de suivre l'évolution des teneurs en $\mathrm{As}, \mathrm{Cd}, \mathrm{Cu}$, Mo, Ni et Zn.

\section{Matériels et méthodes}

\subsection{Caractérisation du sédiment}

Après dragage du sédiment dans la Rade de Toulon, le sédiment a été déposé sur un site pilote avant d'être conditionné pour le transport au laboratoire. La contamination inorganique du sédiment a été déterminée par l'analyse de $\mathrm{As}, \mathrm{Cd}, \mathrm{Cu}, \mathrm{Mo}$, Ni et $\mathrm{Zn}$ en ICP-MS (Spectromètre de masse à plasma inductif). Les organoétains (Triphénylétain (TPhT), Tributylétain (TBT), Dibutylétain (DBT) et Monobutylétain (MBT) ) ont été analysés par chromatographie en phase gazeuse - plasma induit sous champ micro-onde - détection par spectrométrie d'émission atomique (GC-MIP-AED) et les HAPs (HAP totaux $=\sum$ Fluoranthène, Benzo(b)fluoranthène, Benzo(k)fluoranthène, Benzo(a)pyrène, Benzo(ghi)pérylène, Indéno(1,2,3-cd)pyrène) par chromatographie liquide avec détection fluorimétrique.

\subsection{Compostage des sédiments frais}

Le compostage du sédiment a été réalisé en milieu extérieur pendant 3 mois. Le dispositif expérimental se compose de six compartiments dont la base est une grille en plastique avec des mailles de quelques centimètres. En dessous de chaque compartiment recouvert de géotextile, des bacs en polypropylène ont été disposés afin de récupérer les eaux de percolation. Des andains de $6 \mathrm{~kg}$ de sédiment frais ont été placés dans les 6 compartiments, pour l'un des andains aucun additif a été ajouté (sédiment témoin), les 5 autres ont été respectivement traités avec $5 \%$ de fer zéro en poudre, $5 \%$ de clinoptilolite en poudre et grossière $(0.5 \mathrm{~mm}), 5 \%$ et $15 \%$ d'hématite en poudre. 
Le traitement par compostage actif consiste à aérer les 6 andains par retournement manuel et à les humidifier une fois par semaine. Les concentrations totales en $\mathrm{As}, \mathrm{Cd}$, $\mathrm{Cu}, \mathrm{Mo}$, Ni et $\mathrm{Zn}$ dans les lixiviats filtrés ont été déterminées en ICP MS. L'effet du compostage a été évalué en déterminant les concentrations en HAP totaux et organoétains (TPhT, TBT, DBT, MBT) sur le sédiment frais et composté.

\section{Résultats et discussion}

\subsection{Niveau de contamination du sédiment frais}

Les concentrations des polluants (As, $\mathrm{Cd}, \mathrm{Cu}, \mathrm{Ni}$ et $\mathrm{Zn}$, TBT), pour lesquels les niveaux réglementaires $\mathrm{N} 1$ et $\mathrm{N} 2$ existent, sont données dans le Tableau 1. Les concentrations totales en polluants inorganiques et en TBT sont très élevées dans le sédiment étudié. Plusieurs polluants inorganiques sont au dessus du niveau de contamination N2. En effet, la teneur en $\mathrm{Cu}$ est 18 fois supérieure au niveau N2, Zn dépasse de 1200 fois son niveau N2 et la teneur en As est 2.5 fois plus élevée que son niveau N2 réglementaire.

Quant au TBT, sa concentration est supérieure de 20 fois le niveau N2. Les analyses chimiques montrent que le sédiment étudié est fortement contaminé en polluants minéraux et en TBT. Ce sédiment présente donc un risque pour le milieu marin, son immersion en mer sera interdite. Compte tenu de son statut de déchet, il est donc nécessaire d'envisager un traitement efficace afin de réduire la nocivité du déchet pour l'environnement.

Tableau 1. Concentrations totales en polluants inorganiques $(\mathrm{mg} / \mathrm{kg})$ et en TBT ( $\mu \mathrm{g}$ $\mathrm{Sn} / \mathrm{kg}$ ) dans le sédiment fraîchement dragué.

\begin{tabular}{ccccccc}
\hline & $A s$ & $C d$ & $C u$ & $N i$ & $Z n$ & $T B T$ \\
\hline N1 & 25 & 1.2 & 45 & 37 & 276 & 400 \\
N2 & 50 & 2.4 & 90 & 74 & 552 & 1000 \\
Sédiment & 128 & 1.7 & 1582 & 35 & 1723 & 23825 \\
\hline
\end{tabular}

3.2 Stabilisation géochimique par des additifs minéraux

Les concentrations en $\mathrm{As}, \mathrm{Cd}, \mathrm{Cu}, \mathrm{Mo}, \mathrm{Ni}$ et $\mathrm{Zn}$ ont été suivies dans les lixiviats des andains sur une période de 3 mois. A la fin de la période de compostage, les concentrations de ces éléments restent relativement stables malgré les phases d'humidification. A partir des concentrations cumulées au bout des trois mois, des flux de polluants ont été calculés pour le sédiment non traité et les sédiments traités. Les résultats sont exprimés en nmol/kg sédiment et représentés dans la figure 1. Les flux de contaminant issus des sédiments traités sont inférieurs à celui du sédiment témoin excepté pour le sédiment traité avec la clinoptilolite fine. En effet, le sédiment traité à la clinoptilolite fine présente un flux plus important que le sédiment non traité. Ce 
phénomène pourrait s'expliquer par le lessivage et la dissolution des impuretés fixées sur les particules de clinoptilolite. La clinoptilolite présentant une granulométrie plus grossière permet de réduire d'environ $20 \%$ le flux de polluants par rapport au sédiment non traité (témoin). Les additifs à base de fer (hématite et fer zéro) permettent de limiter significativement la dispersion des polluants dans l'environnement en réduisant les flux de métaux de près de 50\% par rapport au témoin. Parmi les polluants étudiés, $\mathrm{Zn}$ et Mo sont majoritairement relargués dans les lixiviats des sédiments stabilisés et du sédiment témoin avec respectivement $14-27 \mathrm{nmol} / \mathrm{kg}$ et $2-18 \mathrm{nmol} / \mathrm{kg}$. Les additifs à base de fer permettent de diminuer respectivement les flux de $\mathrm{Zn}$ et de Mo de $48 \%$ et $80 \%$ dans les lixiviats. Après 3 mois de lixiviation, les pertes en $\mathrm{As}, \mathrm{Cd}, \mathrm{Cu}, \mathrm{Mo}$, Ni et $\mathrm{Zn}$ sont relativement faibles dans le sédiment témoin et représentent respectivement $0.07 \%$, $1.49 \%, 0.03 \%, 5.33 \%, 0.33 \%$ et $0.08 \%$ de leurs concentrations totales.

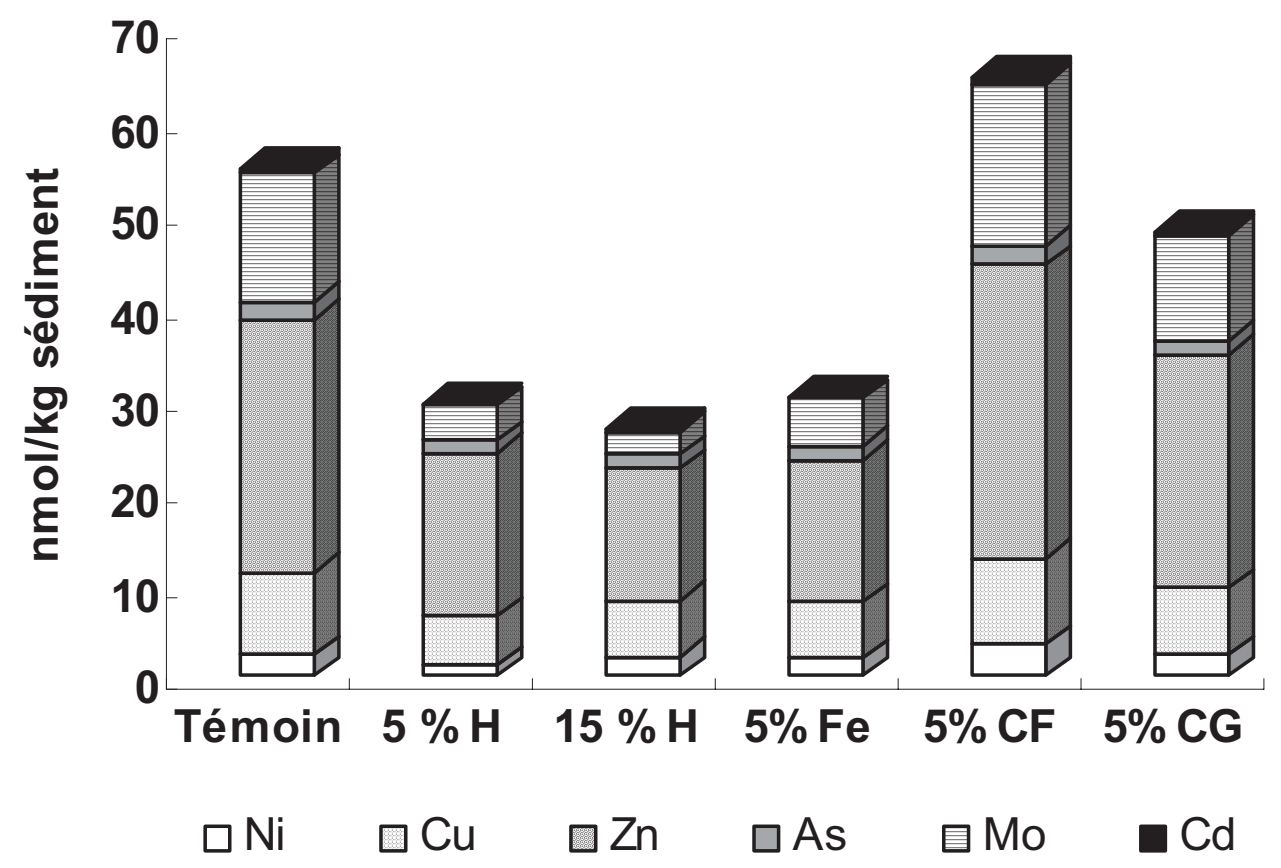

Figure 1. Flux de polluants (nmol/kg sédiment) issus du sédiment non traité (témoin) et des sédiments traités par des additifs minéraux ( $\mathrm{H}$ : Hématite ; Fe : Fer zéro ; $\mathrm{CF}$ :

Clinoptilolite fine ; CG : Clinoptilolite grossière) après 3 mois de compostage. 
3.3 Effet du compostage sur la dégradation des organoétains et des HAPs

Les résultats de l'analyse des polluants organiques avant et après compostage (Tableau 2) indiquent une diminution des concentrations en organoétains et HAPs après compostage. Les organoétains présentent des taux de dégradation élevés entre 38 et $91 \%$ par rapport aux HAP totaux qui présentent uniquement $16 \%$ de dégradation. Ce faible taux de dégradation pourrait s'expliquer par une température de compostage trop faible pour la dégradation des composés à longue chaîne carbonée. Parmi les organoétains, le TPhT et les produits de dégradation du TBT (MBT et DBT) sont beaucoup mieux dégradés que le TBT. D'après SAEKI et al. (2007), la dégradation des organoétains dans les sédiments marins serait liée à l'augmentation de l'activité microbienne lors du compostage actif et non à la photo-dégradation.

\section{Conclusions}

La stabilisation des sédiments contaminés par des additifs minéraux à base de fer (hématite et fer métallique) permet d'améliorer significativement la qualité des eaux de percolation en réduisant de 20 à 50\% les flux de polluants dans les eaux de percolation. Le traitement par compostage des boues de dragages permet d'abattre entre 38 et $91 \%$ de la teneur initiale en organoétains (composés hautement toxique : TBT, TPhT). La stabilisation géochimique associée à un traitement par compostage constitue un procédé intéressant pour l'élimination ou la valorisation des sédiments de dragage contaminés. De plus, ce traitement permettrait une gestion durable et peu onéreuse des sédiments portuaires.

Tableau 2. Teneurs en organoétains $(\mu \mathrm{g} \mathrm{Sn} / \mathrm{kg})$ et HAP totaux $(\mathrm{mg} / \mathrm{kg})$ sur le sédiment frais et le sédiment traité par compostage pendant 3 mois.

\begin{tabular}{llllll}
\hline Polluants & TPhT & TBT & DBT & MBT & HAP totaux \\
& & & & & \\
\hline Sédiment frais & 680 & 23825 & 6522 & 3758 & 38 \\
Sédiment composté & 62 & 14728 & 1413 & 884 & 32 \\
Taux de dégradation (\%) & 91 & 38 & 78 & 75 & 16 \\
\hline
\end{tabular}

\section{Remerciements}

Les auteurs remercient le Conseil Général du Var (Projet SEDIMARD 83) et l'Agence de l'Eau pour le financement de cette étude. 
Meilleures pratiques environnementales en ingénierie côtière et maritime

\section{Références}

ALZIEU C., MICHEL P., CHIFFOLEAU P., BOUTIER B., ABARNOU A. (1999). Dragages et environnement marin. Ifremer, Brest et Nantes. Ministère de l'Aménagement du territoire et de l'Environnement, Paris, pp 27-35. ISBN 2-84433014-2, $233 \mathrm{p}$.

SAEKI K., NABESHIMA A., KUNITOB T., OSHIMA Y. (2007). The stability of butyltin compounds in a dredged heavily-contaminated sediment. Chemosphere, vol. 68, pp 1114-1119. 